\title{
FRACTIONAL POSITIVE CONTINUOUS-TIME LINEAR SYSTEMS AND THEIR REACHABILITY
}

\author{
TADEUSZ KACZOREK
}

Faculty of Electrical Engineering

Białystok Technical University

ul. Wiejska 45D, 15-351 Białystok, Poland

e-mail:kaczorek@isep.pw.edu.pl

\begin{abstract}
A new class of fractional linear continuous-time linear systems described by state equations is introduced. The solution to the state equations is derived using the Laplace transform. Necessary and sufficient conditions are established for the internal and external positivity of fractional systems. Sufficient conditions are given for the reachability of fractional positive systems.
\end{abstract}

Keywords: fractional systems, positive systems, reachability.

\section{Introduction}

In positive systems, inputs, state variables and outputs take only nonnegative values. Examples of positive systems are industrial processes involving chemical reactors, heat exchangers and distillation columns, storage systems, compartmental systems, water and atmospheric pollution models. A variety of models behaving as positive linear systems can be found in engineering, management science, economics, social sciences, biology, medicine, etc.

Positive linear systems are defined on cones and not on linear spaces. Therefore, the theory of positive systems is more complicated and less advanced. An overview of the state of the art in positive systems is given in the monographs (Farina and Rinaldi, 2000; Kaczorek, 2002). An extension of positive systems are cone systems (Kaczorek, 2006; Kaczorek, 2007b).

The notion of cone systems was introduced in (Kaczorek, 2006). Roughly speaking, a cone system is a system obtained from a positive one by substitution of the positive orthants of states, inputs and outputs by suitable arbitrary cones. The realization problem for cone systems was addressed in (Kaczorek, 2006; Kaczorek, 2007a). The positive controllability of dynamical systems was investigated in (Klamka, 2002) and the approximate constrained controllability of mechanical systems in (Klamka, 2005).
The first definition of the fractional derivative was introduced by Liouville and Riemann at the end of the 19-th century (Nishimoto, 1984; Miller and Ross, 1993; Podlubny, 1999). This idea was used by engineers for modelling various processes in the late 1960s (Vinagre et al., 2002; Vinagre and Feliu, 2002; Zaborowsky and Meylaov, 2001). Mathematical fundamentals of fractional calculus are given in the monographs (Miller and Ross, 1993; Nishimoto, 1984; Podlubny, 1999; Oldham and Spanier, 1974; Oustalup, 1993). Fractional-order controllers were developed in (Oustalup, 1993; Podlubny et al. 1997). A generalization of the Kalman filter for fractional-order systems was proposed in (Sierociuk and Dzieliński, 2006). Some others applications of fractionalorder systems can be found in (Engheta, 1997; Ostalczyk, 2000; Ostalczyk, 2004a; Ostalczyk, 2004b; Ferreira and Machado, 2003; Moshrefi-Torbati and Hammond, 1998; Reyes-Melo et al.,2004; Riu et al., 2001; Sjöberg and Kari, 2002; Vinagre et al., 2002; Samko et al., 1993). In (Ortigueira, 1997), a method for computation of the impulse responses from the frequency responses for fractional standard (nonpositive) discrete-time linear systems was given. Fractional polynomials and $n \mathrm{D}$ systems were investigated in (Gałkowski and Kummert, 2005).

In this paper a new class of fractional positive continuous-time systems described by state equations will be introduced, and necessary and sufficient conditions for internal and external positivity will be established. 
The paper is organized as follows: In Section 2, using the Caputo definition and Laplace transform, a solution to the state equations of fractional systems is derived. The necessary and sufficient conditions for the internal and external positivity of fractional systems are established in Section 3. In Section 4, the reachability of positive fractional systems is investigated. Concluding remarks are given in Section 5.

To the best of the author's knowledge, positive fractional continuous-time linear systems have not been considered yet.

The following notation will be used in the paper: the set of $n \times m$ real matrices will be denoted by $\mathbb{R}^{n \times m}$, and $\mathbb{R}^{n}:=\mathbb{R}^{n \times 1}$. The set of $n \times m$ real matrices with nonnegative entries will be denoted by $\mathbb{R}_{+}^{m \times n}$, and $\mathbb{R}_{+}^{n}:=\mathbb{R}_{+}^{n \times 1}$. A matrix $A$ with nonnegative entries will be also denoted by $A \geq 0$. The set of nonnegative integers will be denoted by $\mathbb{Z}_{+}$and the $n \times n$ identity matrix by $I_{n}$.

\section{Continuous-time fractional linear systems and their solutions}

In this paper, the following Caputo definition of the fractional derivative will be used (Oustalup, 1993):

$$
\begin{aligned}
D^{\alpha} f(t)= & \frac{\mathrm{d}^{\alpha}}{\mathrm{d} t^{\alpha}} f(t) \\
= & \frac{1}{\Gamma(n-\alpha)} \int_{0}^{t} \frac{f^{(n)}(\tau)}{(t-\tau)^{\alpha+1-n}} \mathrm{~d} \tau, \\
& n-1<\alpha \leq n \in \mathbb{N}=\{1,2, \ldots\},
\end{aligned}
$$

where $\alpha \in \mathbb{R}$ is the order of the fractional derivative and

$$
f^{(n)}(\tau)=\frac{\mathrm{d}^{n} f(\tau)}{\mathrm{d} \tau^{n}} .
$$

Consider the continuous-time fractional linear system described by the state equations

$$
\begin{aligned}
D^{\alpha} x(t) & =A x(t)+B u(t), \quad 0<\alpha \leq 1, \\
y(t) & =C x(t)+D u(t),
\end{aligned}
$$

where $x(t) \in \mathbb{R}^{N}, u(t) \in \mathbb{R}^{m}, y(t) \in \mathbb{R}^{p}$ are respectively the state, input and output vectors, and $A \in \mathbb{R}^{N \times N}, B \in$ $\mathbb{R}^{N \times m}, C \in \mathbb{R}^{p \times N}, D \in \mathbb{R}^{p \times m}$.

Theorem 1. The solution to $(2 a)$ is given by

$$
\begin{aligned}
& x(t)=\Phi_{0}(t) x_{0}+\int_{0}^{t} \Phi(t-\tau) B u(\tau) \mathrm{d} \tau, \\
& x(0)=x_{0},
\end{aligned}
$$

where

$$
\begin{aligned}
\Phi_{0}(t) & =E_{\alpha}\left(A t^{\alpha}\right)=\sum_{k=0}^{\infty} \frac{A^{k} t^{k \alpha}}{\Gamma(k \alpha+1)}, \\
\Phi(t) & =\sum_{k=0}^{\infty} \frac{A^{k} t^{(k+1) \alpha-1}}{\Gamma[(k+1) \alpha]},
\end{aligned}
$$

$E_{\alpha}\left(A t^{\alpha}\right)$ is the Mittage-Leffler matrix function, $\Gamma(x)=$ $\int_{0}^{\infty} e^{-t} t^{x-1} \mathrm{~d} t$ is the gamma function.

Proof. Applying the Laplace transform to (2a) and taking into account that

$$
\begin{aligned}
\mathcal{L}\left[D^{\alpha} x(t)\right] & =s^{\alpha} X(s)-s^{\alpha-1} x_{0}, \\
X(s) & =L[x(t)]=\int_{0}^{\infty} x(t) e^{-s t} \mathrm{~d} t,
\end{aligned}
$$

we obtain

$$
X(s)=\left[I_{N} s^{\alpha}-A\right]^{-1}\left(s^{\alpha-1} x_{0}+B U(s)\right),
$$

where $U(s)=L[u(t)]$.

It is easy to check that

$$
\left[I_{N} s^{\alpha}-A\right]^{-1}=\sum_{k=0}^{\infty} A^{k} s^{-(k+1) \alpha}
$$

since

$$
\left[I_{N} s^{\alpha}-A\right]\left(\sum_{k=0}^{\infty} A^{k} s^{-(k+1) \alpha}\right)=I_{N} .
$$

Substitution of (8) into (7) yields

$$
\begin{aligned}
& X(s) \\
& =\sum_{k=0}^{\infty} A^{k} s^{-(k \alpha+1)} x_{0}+\sum_{k=0}^{\infty} A^{k} s^{-(k+1) \alpha} B U(s) .
\end{aligned}
$$

Applying the inverse Laplace transformation to (10) and the convolution theorem, we obtain

$$
\begin{aligned}
x(t)= & L^{-1}[X(s)]=\sum_{k=0}^{\infty} A^{k} L^{-1}\left[s^{-(k \alpha+1)}\right] x_{0} \\
& +\sum_{k=0}^{\infty} A^{k} L^{-1}\left[s^{-(k+1) \alpha} B U(s)\right] \\
= & \Phi_{0}(t) x_{0}+\int_{0}^{t} \Phi(t-\tau) B u(\tau) \mathrm{d} \tau
\end{aligned}
$$

where

$$
\begin{aligned}
\Phi_{0}(t) & =\sum_{k=0}^{\infty} A^{k} L^{-1}\left[s^{-(k \alpha+1)}\right]=\sum_{k=0}^{\infty} \frac{A^{k} t^{k \alpha}}{\Gamma(k \alpha+1)} \\
\Phi(t) & =L^{-1}\left\{\left[I_{N} s^{\alpha}-A\right]^{-1}\right\}=\sum_{k=0}^{\infty} A^{k} L^{-1}\left[s^{-(k+1) \alpha}\right] \\
& =\sum_{k=0}^{\infty} \frac{A^{k} t^{(k+1) \alpha-1}}{\Gamma[(k+1) \alpha]}
\end{aligned}
$$


Note that the solution (3) of (2a) for $B u(t)=0$ and $x_{0} \neq 0$ is the same as that in (Vinagre et al., 2002), but the second term of (3) is different.

Remark 1. From (4) and (5) for $\alpha=1$ we have

$$
\Phi_{0}(t)=\Phi(t)=\sum_{k=0}^{\infty} \frac{(A t)^{k}}{\Gamma(k+1)}=e^{A t} .
$$

Remark 2. Note that the classical Cayley-Hamilton theorem yields that if

$$
\begin{aligned}
\operatorname{det} & {\left[I_{N} s^{\alpha}-A\right] } \\
\quad & =\left(s^{\alpha}\right)^{N}+a_{N-1}\left(s^{\alpha}\right)^{N-1}+\cdots+a_{1} s^{\alpha}+a_{0},
\end{aligned}
$$

then

$$
A^{N}+a_{N-1} A^{N-1}+\cdots+a_{1} A+a_{0} I=0 .
$$

Example 1. Find the solution to (2a) for $0<\alpha \leq 1$ and

$$
\begin{gathered}
A=\left[\begin{array}{ll}
0 & 1 \\
0 & 0
\end{array}\right], B=\left[\begin{array}{l}
0 \\
1
\end{array}\right], x_{0}=\left[\begin{array}{l}
1 \\
1
\end{array}\right], \\
u(t)=1(t)= \begin{cases}1 & \text { for } \mathrm{t}>0, \\
0 & \text { for } \mathrm{t}<0 .\end{cases}
\end{gathered}
$$

Using (4) and (5), we obtain

$$
\begin{aligned}
\Phi_{0}(t) & =\sum_{k=0}^{\infty} \frac{A^{k} t^{k \alpha}}{\Gamma(k \alpha+1)}=I_{2}+\frac{A t^{\alpha}}{\Gamma(\alpha+1)}, \\
\Phi(t) & =I_{2} \frac{t^{\alpha-1}}{\Gamma(\alpha)}+A \frac{t^{2 \alpha-1}}{\Gamma(2 \alpha)}
\end{aligned}
$$

since

$$
A^{k}=\left[\begin{array}{ll}
0 & 1 \\
0 & 0
\end{array}\right]^{k}=\left[\begin{array}{ll}
0 & 0 \\
0 & 0
\end{array}\right] \text { for } k=2,3, \cdots
$$

Substitution of (15) and $u(t)=1$ into (3) yields

$$
\begin{aligned}
x(t)= & \Phi_{0}(t) x_{0}+\int_{0}^{t} \Phi(t-\tau) B u(\tau) \mathrm{d} \tau \\
= & x_{0}+\frac{A x_{0} t^{\alpha}}{\Gamma(\alpha+1)} \\
& +\int_{0}^{t}\left(\frac{B}{\Gamma(\alpha)}(t-\tau)^{\alpha-1}+\frac{A B}{\Gamma(2 \alpha)}(t-\tau)^{2 \alpha-1}\right) \mathrm{d} \tau \\
= & x_{0}+\frac{A x_{0} t^{\alpha}}{\Gamma(\alpha+1)}+\frac{B t^{\alpha}}{\Gamma(\alpha+1)}+\frac{A B t^{2 \alpha}}{\Gamma(2 \alpha+1)} \\
= & {\left[\begin{array}{c}
1+\frac{t^{\alpha}}{\Gamma(\alpha+1)}+\frac{t^{2 \alpha}}{\Gamma(2 \alpha+1)} \\
1+\frac{t^{\alpha}}{\Gamma(\alpha+1)}
\end{array}\right] }
\end{aligned}
$$

since $\Gamma(\alpha+1)=\alpha \Gamma(\alpha)$.

\section{Positivity of continuous-time fractional systems}

Definition 1. The fractional system (2) is called an internally positive fractional system if and only if $x(t) \in \mathbb{R}_{+}^{N}$ and $y(t) \in \mathbb{R}_{+}^{p}$ for $t \geq 0$ for any initial conditions $x_{0} \in \mathbb{R}_{+}^{N}$ and all inputs $u(t) \in \mathbb{R}_{+}^{m}, t \geq 0$.

A square real matrix $A=\left[a_{i j}\right]$ is called a Metzler matrix if its off-diagonal entries are nonnegative, i.e. $a_{i j} \geq 0$ for $i \neq j$ (Engheta, 1997; Kaczorek, 2002).

Lemma 1. Let $A \in \mathbb{R}^{N \times N}$ and $0<\alpha \leq 1$. Then

$$
\Phi_{0}(t)=\sum_{k=0}^{\infty} \frac{A^{k} t^{k \alpha}}{\Gamma(k \alpha+1)} \in \mathbb{R}_{+}^{N \times N} \quad \text { for } t \geq 0
$$

and

$$
\Phi(t)=\sum_{k=0}^{\infty} \frac{A^{k} t^{(k+1) \alpha-1}}{\Gamma[(k+1) \alpha]} \in \mathbb{R}_{+}^{N \times N} \quad \text { for } t \geq 0
$$

if and only if $A$ is a Metzler matrix.

Proof. (Necessity) From the expansions

$$
\begin{aligned}
\Phi_{0}(t) & =I_{N}+\frac{A}{\Gamma(\alpha+1)}+\cdots, \\
\Phi(t) & =I_{N} \frac{t^{(\alpha-1)}}{\Gamma(\alpha)}+A \frac{t^{2 \alpha-1}}{\Gamma(2 \alpha)}+\cdots
\end{aligned}
$$

it follows that $\Phi_{0}(t) \in \mathbb{R}_{+}^{N \times N}$ and $\Phi(t) \in \mathbb{R}_{+}^{N \times N}$ for small $t>0$ only if $A$ is a Metzler matrix.

(Sufficiency) It is well known (Kaczorek, 2002) that

$$
e^{A t} \in \mathbb{R}_{+}^{N \times N} \text { for } t \geq 0
$$

if and only if $A$ is a Metzler matrix.

Using (17) we may write

$$
\begin{aligned}
\Phi_{0}(t)-e^{A t^{\alpha}} & =\sum_{k=0}^{\infty}\left(\frac{\left(A t^{\alpha}\right)^{k}}{\Gamma(k \alpha+1)}-\frac{\left(A t^{\alpha}\right)^{k}}{k !}\right) \\
& =\sum_{k=0}^{\infty} \frac{k !-\Gamma(k \alpha+1)}{\Gamma(k \alpha+1)} \frac{\left(A t^{\alpha}\right)^{k}}{k !} \geq 0 \\
& \text { for } t \geq 0(20)
\end{aligned}
$$

since $k ! \geq \Gamma(k \alpha+1)$ for $0<\alpha \leq 1$. Thus from (20) and (19) we have $\Phi_{0}(t) \geq e^{A t^{\alpha}} \geq 0$ for $t \geq 0$. The proof for (18) is similar.

Theorem 2. The continuous-time fractional system (2) is internally positive if and only if the matrix $A$ is a Metzler matrix and

$$
B \in \mathbb{R}_{+}^{N \times M}, \quad C \in \mathbb{R}_{+}^{p \times N}, \quad D \in \mathbb{R}_{+}^{p \times m} .
$$


Proof. (Sufficiency) By Theorem 1 the solution of (2a) has the form (3) and $x(t) \in \mathbb{R}_{+}^{N}, t \geq 0$ if (18) holds and $\mathrm{A}$ is a Metzler matrix since $\Phi_{0}(t) \in \mathbb{R}_{+}^{N \times N}, x_{0} \in \mathbb{R}_{+}^{m}$ and $u(t) \in \mathbb{R}_{+}^{m}$ for $t \geq 0$.

(Necessity) Let $u(t)=0, t \geq 0$ and $x_{0}=e_{i}$ (the $i$-th column of the identity matrix $I_{N}$ ). The trajectory of the system does not leave the orthant $\mathbb{R}_{+}^{N}$ only if $x^{\alpha}(0)=$ $A e_{i} \geq 0$, which implies $a_{i j} \geq 0$ for $i \neq j$. The matrix $A$ has to be a Metzler matrix. For the same reason, for $x_{0}=0$ we have $x^{a}(0)=B u(0) \geq 0$, which implies $B \in \mathbb{R}_{+}^{N \times m}$, since $u(0) \in \mathbb{R}_{+}^{m}$ may be arbitrary. From (2b) for $u(t)=0, t \geq 0$ we have $y(0)=C x_{0} \geq 0$ and $C \in \mathbb{R}_{+}^{p \times N}$, since $x_{0} \in \mathbb{R}_{+}^{N}$ may be arbitrary. In a similar way, assuming $x_{0}=0$, we obtain $y(0)=D u(0) \geq 0$ and $D \in \mathbb{R}_{+}^{p \times m}$, since $u(0) \in \mathbb{R}_{+}^{m}$ may be arbitrary.

Definition 2. The fractional system (2) is called externally positive if and only if $y(t) \in \mathbb{R}_{+}^{p}, t \geq 0$ for every input $u(t) \in \mathbb{R}_{+}^{m}, t \geq 0$ and $x_{0}=0$.

The impulse response $g(t)$ of a single-input singleoutput system is called its output for the input equal to the Dirac impulse $\delta(t)$ with zero initial conditions. Assuming successively that only one input is equal to $\delta(t)$ and the remaining inputs and initial conditions are zero, we may define the impulse response matrix $g(t) \in \mathbb{R}^{p \times m}$ of the system (2).

The impulse response matrix of the system (2) is given by

$$
g(t)=C \Phi(t) B+D \delta(t) \text { for } t \geq 0 .
$$

Substitution of (3) into ( $2 \mathrm{~b}$ ) for $x_{0}=0$ yields

$$
y(t)=\int_{0}^{t} C \Phi(t-\tau) B u(\tau) \mathrm{d} \tau+D u(t), \quad t \geq 0 .
$$

The formula (22) follows from (23) for $u(t)=\delta(t)$.

Theorem 3. The continuous-time fractional system (2) is externally positive if and only if its impulse response matrix (22) is nonnegative, i.e.,

$$
g(t) \in \mathbb{R}_{+}^{p \times m} \quad \text { for } t \geq 0 .
$$

Proof. The necessity of the condition (24) follows immediately from Definition 2. The output $y(t)$ of the system (2) with zero initial conditions for any input $u(t)$ is given by the formula

$$
y(t)=\int_{0}^{t} g(t-\tau) u(\tau) \mathrm{d} \tau,
$$

which can be obtained by the substitution of (22) into (23). If the condition (24) is met and $u(t) \in \mathbb{R}_{+}^{m}$, then from (25) we have $y(t) \in \mathbb{R}_{+}^{p} \quad$ for $t \geq 0$.
From (22) and (18) it follows that if $A$ is a Metzler matrix and (21) holds, then the impulse response matrix (22) is nonnegative. Therefore, we have the following two corollaries:

Corollary 1. The impulse response matrix (22) of the internally positive system (2) is nonnegative.

Corollary 2. Every continuous-time fractional internally positive system (2) is also externally positive.

\section{Reachability}

Definition 3. The state $x_{f} \in \mathbb{R}_{+}^{N}$ of the fractional system (2) is called reachable in time $t_{f}$ if there exist an input $u(t) \in \mathbb{R}_{+}^{m}, \quad t \in\left[0, t_{f}\right]$ which steers the state of (2) from the zero initial state $x_{0}=0$ to $x_{f}$. If every state $x_{f} \in \mathbb{R}_{+}^{N}$ is reachable in time $t_{f}$, the system is called reachable in time $t_{f}$. If for every state $x_{f} \in \mathbb{R}_{+}^{N}$ there exists a time $t_{f}$ such that the state is reachable in time $t_{f}$, then the system (2) is called reachable.

A real square matrix is called monomial if and only if each its row and column contains only one positive entry and the remaining entries are zero.

Theorem 4. The continuous-time fractional system (2) is reachable in time $t_{f}$ if the matrix

$$
R\left(t_{f}\right)=\int_{0}^{t} \Phi(\tau) B B^{T} \Phi^{T}(\tau) \mathrm{d} \tau
$$

is a monomial matrix. The input which steers the state of the system (2) from $x_{0}=0$ to $x_{f}$ is given by

$$
u(t)=B^{T} \Phi^{T}\left(t_{f}-t\right) R^{-1}\left(t_{f}\right) x_{f},
$$

where $T$ denotes the transpose.

Proof. If the matrix (26) is a monomial matrix, then $R^{-1}\left(t_{f}\right) \in \mathbb{R}_{+}^{N \times N}$ and the input defined by (27) is a nonnegative vector, i.e. $u(t) \in \mathbb{R}_{+}^{m}, \quad t \geq 0$. Using (3) for $x_{0}=0, t=t_{f},(27)$ and (26) we obtain

$$
\begin{aligned}
x\left(t_{f}\right) & =\int_{0}^{t_{f}} \Phi\left(t_{f}-\tau\right) B B^{T} \Phi^{T}\left(t_{f}-\tau\right) \mathrm{d} \tau R^{-1}\left(t_{f}\right) x_{f} \\
& =\int_{0}^{t_{f}} \Phi(\tau) B B^{T} \Phi^{T}(\tau) \mathrm{d} \tau R^{-1}\left(t_{f}\right) x_{f}=x_{f} .
\end{aligned}
$$

Therefore, the input (27) steers the state of the system (2) from $x_{0}=0$ to $x_{f}$.

Theorem 5. If $A=\operatorname{diag}\left[a_{1}, a_{2}, \ldots, a_{N}\right] \in \mathbb{R}_{+}^{N \times N}$ and $B \in \mathbb{R}_{+}^{N \times m}$ is a monomial matrix, then the continuoustime fractional system (2) is reachable. 
Proof. From (5) it follows that if the matrix A is diagonal, then so is the matrix $\Phi(t)$ and the matrix $\Phi(t) B$ is monomial since, by assumption, the matrix $B$ is monomial. From (26) written in the form

$$
R\left(t_{f}\right)=\int_{0}^{t_{f}} \Phi(\tau) B[\Phi(\tau) B]^{T} \mathrm{~d} \tau
$$

it follows that the matrix (28) is monomial. Thus, by Theorem 3 , the fractional system is reachable.

Example 2. We shall show that the fractional system (2) with

$$
A=\left[\begin{array}{ll}
1 & 0 \\
0 & 0
\end{array}\right], \quad B=\left[\begin{array}{ll}
0 & 1 \\
1 & 0
\end{array}\right]
$$

is reachable. Taking into account that

$$
A^{k}=\left[\begin{array}{ll}
1 & 0 \\
0 & 0
\end{array}\right]^{k}=\left[\begin{array}{ll}
1 & 0 \\
0 & 0
\end{array}\right] \text { for } k=1,2, \ldots
$$

and using (5), we obtain

$$
\Phi(t)=\sum_{k=0}^{\infty} \frac{A^{k} t^{(k+1) \alpha-1}}{\Gamma[(k+1) \alpha]}=\left[\begin{array}{cc}
\Phi_{1}(t) & 0 \\
0 & \Phi_{2}(t)
\end{array}\right]
$$

where

$$
\Phi_{1}(t)=\sum_{k=0}^{\infty} \frac{t^{(k+1) \alpha-1}}{\Gamma[(k+1) \alpha]}, \quad \Phi_{2}(t)=\frac{t^{\alpha-1}}{\Gamma(\alpha)}
$$

and

$$
\Phi(t) B=\left[\begin{array}{cc}
0 & \Phi_{1}(t) \\
\Phi_{2}(t) & 0
\end{array}\right]
$$

In this case, from (28) we have

$$
\begin{aligned}
R\left(t_{f}\right) & =\int_{0}^{t_{f}} \Phi(\tau) B[\Phi(\tau) B]^{T} \mathrm{~d} \tau \\
& =\int_{0}^{t_{f}}\left[\begin{array}{cc}
\Phi_{1}^{2}(\tau) & 0 \\
0 & \Phi_{2}^{2}(\tau)
\end{array}\right] \mathrm{d} \tau .
\end{aligned}
$$

The matrix (31) is monomial and by Theorem 3 the fractional system is reachable.

Remark 3. It is well known that the system

$$
\dot{x}=A x+B u
$$

with

$$
A=\left[\begin{array}{ccccc}
0 & 0 & \ldots & 0 & a_{0} \\
1 & 0 & \ldots & 0 & a_{1} \\
0 & 1 & \ldots & 0 & a_{2} \\
\ldots & \ldots & \ldots & \ldots \\
0 & \ldots & \ldots & 1 & a_{N-1}
\end{array}\right], \quad B=\left[\begin{array}{l}
1 \\
0 \\
0 \\
0 \\
0
\end{array}\right]
$$

is reachable for any values of the coefficients $a_{i}, i=$ $0,1, \ldots, N-1$, since the reachability matrix is

$$
\left[B, A B, \cdots, A^{N-1} B\right]=I_{N} .
$$

The system (32) is also reachable as a positive system if $a_{i} \geq 0, i=0,1, \ldots, N-2$. The fractional system (2) with (33) is reachable even for $a_{i}=0, i=0,1, \ldots, N-$ 1 if and only if there exist $u(t)>0, t \in\left[0, t_{f}\right]$ such that the following condition is met:

$$
x_{f}=\int_{0}^{t_{f}}\left[\begin{array}{c}
\frac{\left(t_{f}-\tau\right)^{\alpha-1}}{\Gamma(\alpha)} \\
\frac{\left(t_{f}-\tau\right)^{2 \alpha-1}}{\Gamma(2 \alpha)} \\
\ldots \ldots \ldots \cdots \\
\frac{\left(t_{f}-\tau\right)^{N \alpha-1}}{\Gamma(N \alpha)}
\end{array}\right] u(\tau) \mathrm{d} \tau .
$$

The condition (35) follows from (3) for $x_{0}=0,(34)$ and that for $a_{i}=0, i=0,1, \ldots, N-1$, we have $A^{k}=0$ for $k=N, N+1, \ldots$ and

$$
\Phi(t)=\sum_{k=0}^{N-1} \frac{A^{k} t^{(k+1) \alpha-1}}{\Gamma[(k+1) \alpha]} .
$$

This example shows that the reachability conditions for the fractional positive system (2) are much stronger than the conditions for the positive system (2).

\section{Concluding remarks}

A new class of fractional positive continuous-time systems was introduced. The solution to the state equation describing the fractional systems was derived using the Laplace transform (Theorem 1). The classical CayleyHamilton theorem was extended to fractional systems (Remark 2). Necessary and sufficient conditions were established for the internal and external positivity of fractional systems (Theorems 2 and 3). Sufficient conditions for fractional positive systems are much stronger than for classical positive systems. The deliberations were illustrated by examples of fractional continuous-time linear systems. The deliberations presented for reachability can be extended to the controllability of fractional continuoustime systems. 


\section{Acknowledgement}

This work was supported by the Ministry of Science and Higher Education in Poland under the grant no. NN514 193933.

\section{References}

Engheta N. (1997). On the role of fractional calculus in electromagnetic theory, IEEE Transactions on Atennas and Propagation 39(4): $35-46$.

Farina L.and Rinaldi S.(2000). Positive Linear Systems, Theory and Applications, J. Wiley, New York.

Ferreira N.M.F. and Machado J.A.T. (2003). Fractional-order hybrid control of robotic manipulators, Proceedings of the 11th International Conference on Advanced Robotics ICAR'2003, Coimbra, Portugal, pp. 393-398.

Gałkowski K. and Kummert A. (2005). Fractional polynomials and $\mathrm{nD}$ systems, Proceedings of the IEEE International Symposium on Circuits and Systems, ISCAS'2005, Kobe, Japan, CD-ROM.

Kaczorek T. (2002). Positive $1 D$ and $2 D$ Systems, SpringerVerlag, London.

Kaczorek T. (2006). Computation of realizations of discretetime cone systems, Bulletin of the Polish Academy of Sciences: Technical Sciences 54(3): 347-350.

Kaczorek T. (2007a). Reachability and controllability to zero of positive fractional discrete-time systems, Machine Intelligence and Robotic Control 6(4), (in press).

Kaczorek T.(2007b). Reachability and controllability to zero of cone fractional linear systems, Archives of Control Sciences 17(3): 357-367.

Klamka J. (2002). Positive controllability of positive dynamical systems, Proceedings of American Control Conference, ACC-2002, Anchorage, AL, CD-ROM.

Klamka J. (2005). Approximate constrained controllability of mechanical systems, Journal of Theoretical and Applied Mechanics 43(3): 539-554.

Miller K.S. and B. Ross (1993). An Introduction to the Fractional Calculus and Fractional Differenctial Equations, Willey, New York.

Moshrefi-Torbati M. and K. Hammond (1998). Physical and geometrical interpretation of fractional operators, Journal of the Franklin Institute 335B(6): 1077-1086.

Nishimoto K. (1984). Fractional Calculus, Koriyama: Decartes Press.

Oldham K.B. and J. Spanier (1974). The Fractional Calculus, New York: Academic Press.

Ortigueira M.D. (1997). Fractional discrete-time linear systems, Procedings of the IEEE International Conference on Acoustics, Speech and Signal Processing, Munich, Germany, Vol. 3, pp. 2241-2244.

Ostalczyk P. (2000). The non-integer difference of the discretetime function and its application to the control system synthesis, International Journal of Systems Science 31(12): 1551-1561.
Ostalczyk P. (2004a). Fractional-order backward difference equivalent forms Part I - Horner's form, IFAC Workshop on Fractional Differentation and its Applications, FDA'04, Bordeaux, France, pp. 342-347.

Ostalczyk P. (2004b), Fractional-order backward difference equivalent forms Part II - Polynomial Form. Proceedings the 1st IFAC Workshop Fractional Differentation and its Applications, FDA'04, Bordeaux, France, pp. 348-353.

Oustalup A. (1993). Commande CRONE, Paris, Hermès.

Oustalup A. (1995). La dérivation non entiére, Paris: Hermès.

Podlubny I. (1999). Fractional Differential Equations, San Diego: Academic Press.

Podlubny I. (2002). Geometric and physical interpretation of fractional integration and fractional differentation, Fractional Calculs and Applied Analysis 5(4): 367-386.

Podlubny I., L. Dorcak and I. Kostial (1997). On fractional derivatives, fractional order systems and $\mathrm{PI}^{\lambda} \mathrm{D}^{\mu}$-controllers, Procedings of the 36th IEEE Conference on Decision and Control, San Diego, CA, USA, pp. 4985-4990.

Reyes-Melo M.E., J.J. Martinez-Vega, C.A. Guerrero-Salazar and U. Ortiz-Mendez (2004). Modelling and relaxation phenomena in organic dielectric materials. Application of differential and integral operators of fractional order, $\mathrm{JO}_{\mathrm{O}}$ urnal of Optoelectronics and Advanced Materials 6(3): 1037-1043.

Riu D., N. Retiére and M. Ivanes (2001). Turbine generator modeling by non-integer order systems, Proceedings of the IEEE International Conference on Electric Machines and Drives, IEMDC 2001, Cambridge, MA, USA, pp. 185-187.

Samko S. G., A.A. Kilbas and O.I. Marichev (1993). Fractional Integrals and Derivatives. Theory and Applications. London: Gordon and Breach.

Sierociuk D. and D. Dzieliński (2006). Fractional Kalman filter algorithm for the states, parameters and order of fractional system estimation, International Journal of Applied Mathematics and Computer Science 16(1): 129-140.

Sjöberg M. and L. Kari (2002). Non-linear behavior of a rubber isolator system using fractional derivatives, Vehicle System Dynamics 37(3): 217-236.

Vinagre M., C. A. Monje and A.J. Calderon (2002). Fractional order systems and fractional order control actions. Lecture 3 IEEE CDC'02 TW\#2: Fractional Calculus Applications in Automatic Control and Robotics.

Vinagre M. and V. Feliu (2002) Modeling and control of dynamic systems using fractional calculus: Application to electrochemical processes and flexible structures, Proceedings of the 41st IEEE Conference Decision and Control, Las Vegas, NV, USA, pp. 214-239.

Zaborowsky V. and R. Meylaov (2001). Informational network traffic model based on fractional calculus, Proceedings of the International Conference Info-tech and Info-net, ICII 2001, Beijing, China, Vol. 1, pp. 58-63.

Received: 27 November 2007 Revised: 2 February 2008 